\title{
An Efficient Backoff Scheme for IEEE 802.11 DCF
}

\author{
Ho-Jin Shin, Dong-Ryeol Shin ${ }^{1}$, and Hee-Yong Youn \\ School of Information and Communication Engineering, \\ Sungkyunkwan University, \\ 300 cheoncheon-dong, jangan-gu, suwon, Korea \\ \{hjshin, drshin, youn\}@ece.skku.ac.kr
}

\begin{abstract}
This paper proposes a new backoff algorithm for a CSMA/CA protocol and an analytical model computing the saturation throughput and delay of the scheme. The scheme differs from the standard backoff schemes in that the backoff time is not uniformly chosen in the contention window interval which results in reduced collision probability under high loads. Numerical analysis shows that saturation throughput and delay of the proposed scheme outperform the earlier approach.
\end{abstract}

\section{Introduction}

In IEEE 802.11 carrier sensing is performed both at the physical layer and MAC layer, which is also referred to as physical carrier sensing and virtual carrier sensing, respectively. The PCF is a polling-based protocol, which was designed to support collision free and real time services. This paper focuses on the performance and delay analysis and modeling of a new scheme for DCF in IEEE 802.11 wireless LAN.

There are two techniques used for packet transmission in DCF. The default one is a two-way handshaking mechanism called basic access method. The optional one is a four-way handshaking mechanism, which uses RTS/CTS frame to reserve the channel before data transmission. This mechanism has been introduced to reduce performance degradation due to hidden terminals. However, it has some drawback of increased overhead for short data frames.

According to the CSMA/CA protocol, if the medium is busy, the station has to wait until the end of the current transmission. It then waits for an additional DIFS time and then generates a random backoff interval before transmitting a frame. The backoff counter is decremented as long as the channel is sensed idle. The station transmits a frame when the backoff time counter reaches zero. The initial value of the backoff counter is randomly chosen in the contention window $(0, w-1)$. At the first attempt, $w$ $=\mathrm{W}_{0}$, the minimum size of the contention window. After each unsuccessful transmission, $w$ is doubled, up to a maximum value within which the backoff counter value is again randomly selected with uniform distribution.

As more stations are added, the new contention window size due to collisions is doubled and chosen randomly in $(0, w-1)$. In such a case, there is a good chance for the collided station to select smaller value and thus increasing the possibilities of

${ }^{1}$ This paper was supported by Faculty Research Fund from Sungkyunkwan University. 
collisions with existing nodes with small values of backoff counters, which results in degrading performance.

With this observation in mind, we propose a new scheme deciding the contention window size and procedure decrementing the backoff counter, which gives preferences to the active nodes over colliding nodes to reduce the collision probability and thereby improving the throughput. This is the main contribution of the paper, which is to be detailed later.

In recent year, modeling of IEEE 802.11 has been a research focus since the standard has been proposed. In the literature, performance evaluation of IEEE 802.11 has been carried out either by means of simulation [3][12][13] or analytical models with simplified backoff rule assumptions. In particular, constant or geometrically distributed backoff window was used in [4][5], while [6] considered an exponential backoff limited to two stages by employing a two dimensional Markov chain analysis. This paper investigates the performance of the new proposed scheme in terms of throughput and delay. Then comparing with other models, we demonstrate the effect of the proposed mechanism on network performance.

The rest of the paper is organized as follows. Section 2 briefly describes the DCF of IEEE 802.11 MAC protocols and an analytical model computing the saturated throughput and delay of 802.11 is proposed. Section 3 evaluates the proposed scheme and compares it with earlier models. Finally, Section 4 concludes the paper.

\section{Analysis of DCF}

In this paper we concentrate on the analytical evaluation of the saturation throughput and delay, which is described in [7][8][10][11][12][14]. These fundamental performance figures are defined as the throughput limit reached by the system as the offered load increases, and the corresponding delay with the load that the system can carry in stable condition.

One of key contributions of this paper is to derive an analytical model of the saturation throughput and delay, in the assumption of ideal channel conditions (i.e., no hidden terminals and capture [9]). In addition, the Markov model in [8] does not consider the frame retries limits, and thus it may overestimate the throughput of IEEE 802.11. With this observation, our analysis is based on a more exact Markov chain model [11] taking into the consideration of the maximum frame retransmission limit. We assume a fixed number of wireless stations, each always having a packet available for transmission. In other words, the transmission queue of each station is assumed to be always nonempty. To ease comparison with the model in [11], we use the same symbols and variables as in [11]. The analysis is divided into two parts. First, we examine the behavior of a single station with a Markov model, and obtain the stationary probability $\tau$ that the station transmits a packet in a generic slot time. This probability does not depend on the access mechanism employed as shown in [8]. Then, we express the throughput and delay of both Basic and RTS/CTS access method as a function of the computed value $\tau$. 


\subsection{Markov Chain Model}

We employ the same assumptions in [11] for our analysis. Consider a fixed number $n$ of contending stations. In saturation condition, each station has a packet immediately available for transmission after the completion of each successful transmission. Moreover, being all packets consecutive, each packet needs to wait for a random backoff time before transmission.

Let $b(t)$ be the stochastic process representing the backoff time counter for a given station at slot time $t$. A discrete and integer time scale is adopted: $t$ and $t+1$ correspond to the beginning of two consecutive slot times and the backoff time counter of each station decrements at the beginning of each slot time [8]. Let $s(t)$ be the stochastic process representing the backoff stage $(0, \ldots, m)$ of the station at slot time $t$. As in [8][11], the key conjecture in this model is that the probability $p$ that a transmitted packet collides is independent on the stage $s(t)$ of the station. Thus, the bi-dimensional process $\{s(t), b(t)\}$ is a discrete-time Markov chain, which is shown in Fig. 1.

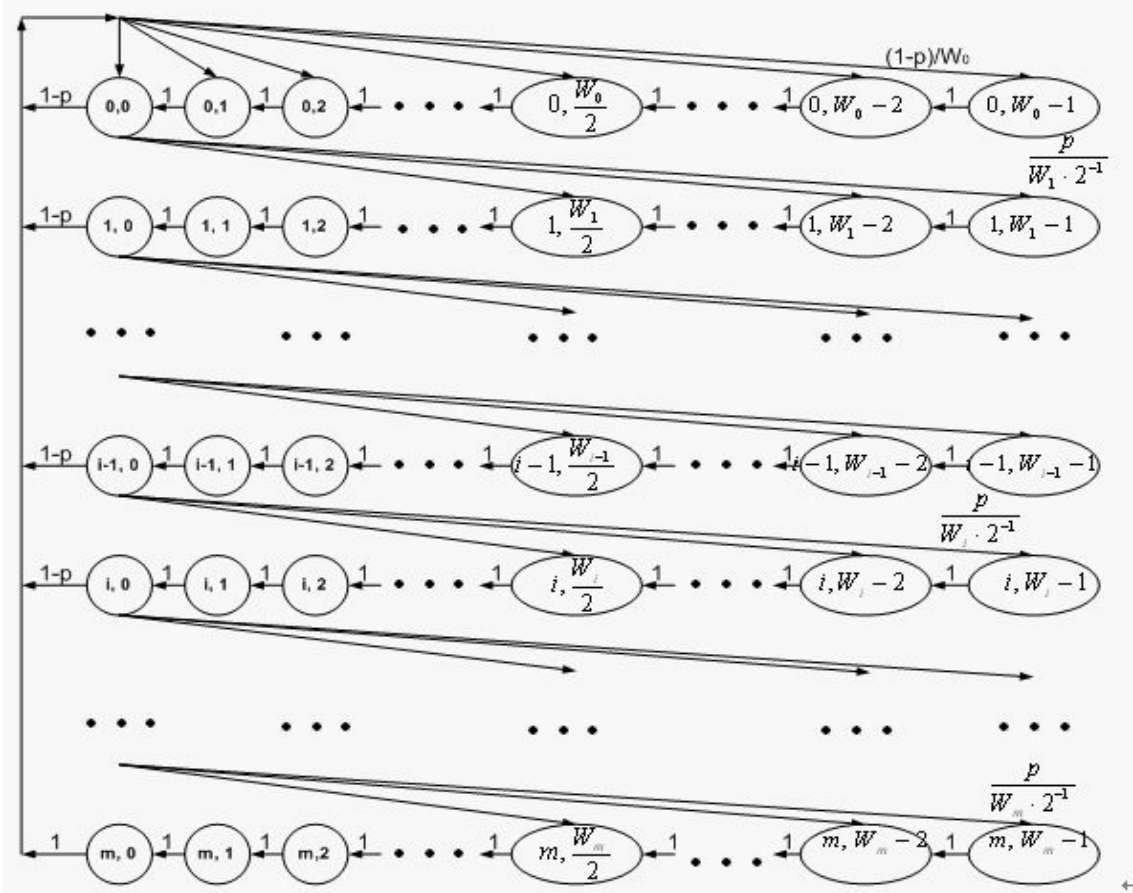

Fig. 1. The Markov Chain model of the new backoff window scheme.

This paper uses all the parameters assigned for Direct Sequence Spread Spectrum (DSSS) PHY in 802.11, for the comparison with the model in [11]. In DSSS, $C W_{\min }$ and $C W_{\max }$ are set to 31 and 1023 , respectively. Therefore, we have 


$$
\left\{\begin{array}{l}
W_{i}=2^{i} W \quad i \leq m^{\prime} \\
W_{i}=2^{m^{\prime}} W \quad i>m^{\prime}
\end{array}\right.
$$

Where $W=\left(C W_{\min }+1\right)$, and $2^{m^{\prime}} W=\left(C W_{\max }+1\right)$. Thus for DSSS, we have $m^{\prime}=5$.

In the IEEE 802.11 backoff procedure, backoff time is randomly chosen between $\left[0, W_{i}-1\right]$, where $W_{i}$ is a contention window at backoff stage- $i$. Fig. 2 and Fig. 3 show the configuration of backoff counter for backoff stage $i=0$ and $i=1$, respectively. This scheme is to reduce the possibility of collision by letting each station get a different range of contention window. The motivation behind choosing a nonuniformly distributed contention window counter value is as follows. For example, a station which transmits a packet successfully generates new backoff time counter value from $\mathrm{CW}_{0}(0 \sim 31)$. If a station cannot transmits a packet due to collision, it moves to the next backoff stage and generates a backoff time counter value from $\mathrm{CW}_{1}(0 \sim 63)$ as shown in [1][8][11]. As a result, there is still a good chance for two stations having the same backoff time counters in which collision may occur when counter reaches zero again since the window size values are integers and multiple of some basic time slot value.

We thus propose new backoff time counter selection scheme. Unlike the schemes in [1][8][11], we give preferences to the stations actively competing with the colliding stations within current contention window for it, the contention window is constructed non-uniformly as follows:

$$
C W_{i}=\left[R_{i}, W_{i}-1\right] \quad 0 \leq i \leq m
$$

Here, if a station transmits a packet successfully in backoff stage- $0, R_{0}=0$. Otherwise, there is a busy channel to incur collision and thus sets a contention window to $\left[W_{i} / 2\right.$, $\left.W_{i}-1\right]$ rather than $\left[0, W_{i}-1\right]$. In other words,

$$
R_{i}=W_{i} / 2 \quad l \leq i \leq m
$$

Comparing with the example above, the proposed scheme adapts a different mechanism for selecting backoff time counter value. The station then moves to the next backoff stage, generating backoff time counter from $\mathrm{CW}_{1}$ (32 63). This approach will reduce the probability of each station choosing the same backoff time counter value. Fig. 4 shows exponential increment of $\mathrm{CW}$ for the proposed backoff time counter scheme.

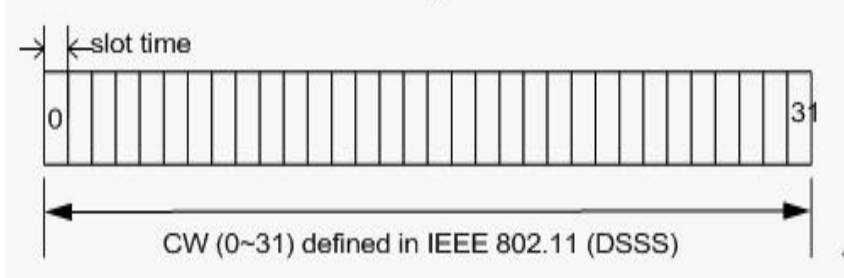

Fig. 2. $C W_{i}$ when backoff stage $i=0$. 


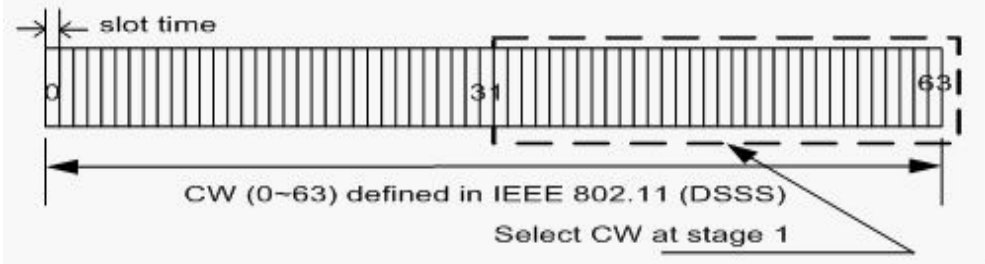

Fig. 3. $C W_{i}$ when backoff stage $i=1$

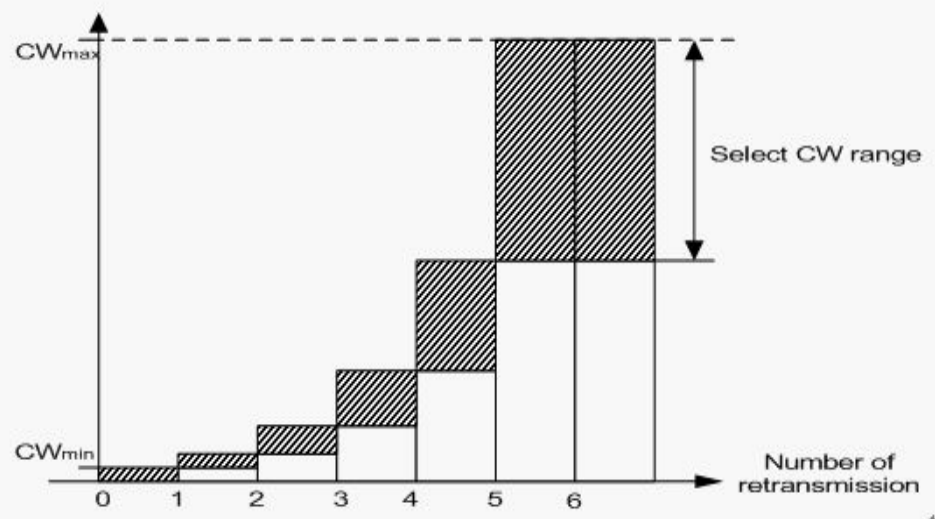

Fig. 4. The backoff counter range with the proposed scheme.

In order to validate and analyze the proposed scheme, we employ the same Markov chain models and assumptions in [11].

Here, the only non-null one-step transition probabilities are

$$
\left\{\begin{array}{l}
P\{i, k \mid i, k+1\}=1 \quad k \in\left(0, W_{i}-2\right) \quad i \in(0, m) \\
P\{0, k \mid i, 0\}=(1-p) / W_{0} \quad k \in\left(0, W_{0}-1\right) \quad i \in(0, m) \\
P\{i, k \mid i-1,0\}=p /\left(W_{i} / 2\right) \quad k \in\left(W_{i} / 2, W_{i}-1\right) \quad i \in(1, m) \\
P\{0, k \mid m, 0\}=1 / W_{0} \quad k \in\left(0, W_{0}-1\right)
\end{array}\right.
$$

The first equation in (2) accounts for the fact that, at the beginning of each slot time, the backoff time is decremented. The second equation accounts for the fact that a new packet following a successful packet transmission starts with backoff stage- 0 . In particular, as considered in the third equation of (2), when an unsuccessful transmission occurs at backoff stage- $(i-1)$, the backoff stage number increases, and the new initial backoff value is randomly chosen in the range $\left(W_{i} / 2, W_{i}-1\right)$. This is different part from others in [8][10][11]. Finally, the fourth case models the fact that when the backoff stage reaches the maximum backoff stage, the contention window is reset if the transmission is unsuccessful or restart the backoff stage for new packet if the transmission is successful. 
The stationary distribution of the Markov chain is defined as $b_{i, k}=\lim _{t \rightarrow \infty} P\{s(t)=i, b(t)=k\}$. Using chain regularities in steady state, we can derive the following relations.

$$
\begin{gathered}
b_{i-1,0} * p=b_{i, 0} \quad 0<i \leq m \\
b_{i, 0}=p^{i} b_{i, 0} \quad 0 \leq i \leq m
\end{gathered}
$$

If a backoff time value $k$ of a station at stage- $i$ is selected among $C W_{i}=\left[W_{i} / 2, W_{i}-1\right]$, the following relation can be derived.

$\left\{b_{i, k}=\frac{W_{i}-k}{W_{i}}(1-p) \sum_{j=0}^{m-1} b_{j, 0}+b_{m, 0} \quad i=0, k \in\left(0, W_{i}-1\right)\right.$

for each $k \in\left(0, \frac{W_{i}}{2}-1\right)$

$b_{i, k}=p b_{i-1,0} \quad 0<i \leq m$

each $k \in\left(\frac{W_{i}}{2}, W_{i}-1\right)$

$b_{i, k}=\frac{W_{i}-k}{W_{i} / 2} p b_{i-1,0} \quad 0<i \leq m$

With (4) and transitions in the chain, equation (5) can be simplified as

$$
b_{i, k}=\gamma^{*} b_{i, 0} \quad 0 \leq i \leq m
$$

By using the normalization condition for stationary distribution, we have

$$
\begin{aligned}
& 1=\sum_{i=0}^{m} \sum_{k=0}^{W_{i}-1} b_{i, k}=\sum_{i=0}^{m} \sum_{k=0}^{W_{i}-1} \gamma b_{i, 0}=\sum_{i=0}^{m} b_{i, 0} \sum_{k=0}^{W_{i}-1} \gamma \\
& =b_{0,0} \sum_{k=0}^{W_{i}-1} \gamma+\sum_{i=1}^{m} b_{i, 0} \sum_{k=0}^{W_{i}-1} \gamma=b_{0,0} \cdot \frac{W_{0}+1}{2}+\sum_{i=1}^{m} b_{i, 0} \sum_{k=0}^{W_{i}-1} \gamma
\end{aligned}
$$

Let $\tau$ be the probability that the station transmits a packet during a generic slot time. A transmission occurs when the backoff window is equal to zero. It is expressed as:

$$
\tau=\sum_{i=0}^{m} b_{i, 0}=\frac{1-p^{m+1}}{1-p} b_{0,0}
$$

Then, using equation (1), (6) and (7), $b_{0,0}$ is computed as 


$$
\begin{aligned}
& b_{0,0}= \begin{cases}\frac{4(1-2 p)(1-p)}{\alpha} & m \leq m^{\prime} \\
\frac{4(1-2 p)(1-p)}{\beta} & m>m^{\prime}\end{cases} \\
& \alpha=2(W+1)(1-2 p)(1-p)+3 W 2 p\left(1-(2 p)^{m}(1-p)+p\left(1-p^{m}\right)(1-2 p)\right. \\
& \beta=2(W+1)(1-2 p)(1-p)+3 W 2 p\left(1-(2 p)^{m^{\prime}}(1-p)+p\left(1-p^{m}\right)(1-2 p)\right. \\
& +3 W 2 m^{\prime} p^{m^{\prime}+1}\left(1-p^{m-m^{\prime}}\right)(1-2 p)
\end{aligned}
$$

In the stationary state, a station transmits a packet with probability $\tau$, so we have

$$
p=1-(1-\tau)^{n-1}
$$

Therefore, equation (8), (9) and (10) represent a nonlinear system with two unknowns $\tau$ and $p$, which can be solved using numerical method. We must have $\tau \in(0,1)$ and $p \in(0,1)$.

Since the Markov chain of Fig. 1 is a little different from that of [8] and [11], the result obtained for $b_{0,0}$ is different from that in [8] and [11]. Similarly, different $\tau$ and $p$.

\subsection{Throughput Analysis}

Once $\tau$ is known, the probability $P_{t r}$ that there is at least one transmission in a slot time given $n$ wireless stations, and the probability $P_{s}$ that a transmission is successful are readily obtained as

$$
\begin{gathered}
P_{t r}=1-(1-\tau)^{n} \\
P_{s}=\frac{n \tau(1-\tau)^{n-1}}{P_{t r}}=\frac{n \tau(1-\tau)^{n-1}}{1-(1-\tau)^{n}}
\end{gathered}
$$

We are finally in the position to determine the normalized system throughput, $S$, defined as the fraction of time the channel is used to successfully transmit payload bits, and express it as a fraction of a slot time

$$
\begin{aligned}
S & =\frac{E \text { [payload infomation transmitted in a slot time }]}{E[\text { length of a slot time }]} \\
& =\frac{P_{s} P_{t r} E[P]}{\left(1-P_{t r}\right) \sigma+P_{s} P_{t r} T_{s}+\left(1-P_{s}\right) P_{t r} T_{c}}
\end{aligned}
$$

Where $E[P]$ is the average packet length, $T_{s}$ is the average time the channel is sensed busy because of a successful transmission, $T_{c}$ is the average time the channel is 
sensed busy by the stations during a collision, and $\sigma$ is the duration of an empty slot time. The times $E[P], T_{s}$ and $T_{c}$ must be measured in slot times.

Let packet header be $H=P H Y_{h d r}+M A C_{h d r}$ and propagation delay be $\delta$. Then we must have the following expression for ACK timeout effect, which are same as in [11].

$$
\left\{\begin{array}{l}
T_{s}^{\text {bas }}=D I F S+H+E[P]+\delta+S I F S+A C K+\delta \\
T_{c}^{\text {bas }}=D I F S+H+E\left[P^{*}\right]+S I F S+A C K
\end{array}\right.
$$

Where bas means basic access method and $E\left[P^{*}\right]$ is the average length of the longest packet payload involved in a collision. In our cases, all the packets have the same fixed size, $E[P]=E\left[P^{*}\right]=P$.

For the RTS/CTS access method,

$$
\left\{\begin{aligned}
T_{s}^{r t s}= & D I F S+R T S+S I F S+\delta+C T S+S I F S+\delta \\
& +H+E[P]+\delta+S I F S+A C K+\delta \\
T_{c}^{r t s}= & D I F S+R T S+S I F S+C T S
\end{aligned}\right.
$$

We suppose collision occurs only between RTS frames and consider only the CTS timeout effect.

\subsection{Delay Analysis}

The delay discussed here refers to the medium access delay; it includes the total time from a station beginning to contend the channel for a transmission to the data frame being transmitted successfully. The delay represents the interval during which two contiguous data frames in a station are transmitted successfully.

Let $D$ be the delay defined above. Then $D$ is computed as

$$
D=T_{s}+D_{s}+D_{c}+T_{s l o t}
$$

In equation (16), $T_{s}$ is the time for a successful transmission, $D_{s}$ is the average time the channel is sensed busy because of successful transmission by other stations, $D_{c}$ is the average time the channel is sensed busy because of collisions, $T_{\text {slot }}$ is the total time of idle slots, which includes the total backoff time of successful transmissions and collisions by each station.

During the interval of two contiguous successful transmissions in a station, the time for a successful transmission in each other station is $T_{s} N_{s}$, where $N_{s}$ is the number of successful transmissions by other stations. For a long enough period time, each station transmits data frame successfully with the same probability. Therefore, during two contiguous successful transmissions in one station, each station must have a successful transmission. We have $N_{s}=n-1$. Then we obtain

$$
D_{s}=T_{s}(n-1)
$$

Let $P_{c}$ be the probability that a collision occurs on the channel, which is 


$$
P_{c}=1-P_{s}
$$

Let $N_{c}$ be the number of contiguous collisions, and we have

$$
P\left\{N_{c}=i\right\}=P_{c}^{i} P_{s}=\left(1-P_{s}\right)^{i} P_{s} \quad i=0,1,2, \cdots .
$$

The mean of $N_{c}$ is

$$
E\left[N_{c}\right]=\sum_{i} i P\left\{N_{c}=i\right\}=\frac{1-P_{s}}{P_{s}}=\frac{1-(1-\tau)^{n}-n \tau(1-\tau)^{n-1}}{n \tau(1-\tau)^{n-1}}
$$

Now consider the whole network. There are $E\left[N_{c}\right]$ contiguous collisions between two random contiguous successful transmissions. According to the analysis above, there are $n$ successful transmissions in the period of a time $D$. Therefore, we have,

$$
D_{c}=n E\left[N_{c}\right] T_{c}=\frac{1-(1-\tau)^{n}-n \tau(1-\tau)^{n-1}}{\tau(1-\tau)^{n-1}} T_{c}
$$

Let $N_{\text {slot }}$ be the number of contiguous idle slots in a backoff interval, then

$$
P\left\{N_{\text {slot }}=i\right\}=\left(1-P_{t r}\right)^{i} P_{t r} \quad i=0,1,2, \cdots .
$$

The mean of $N_{\text {slot }}$ is

$$
E\left[N_{s l o t}\right]=\frac{1-P_{t r}}{P_{t r}}=\frac{(1-\tau)^{n}}{1-(1-\tau)^{n}}
$$

Since there is a backoff interval before each successful transmission or collision and $n$ successful transmissions and $N_{c}$ collisions in the period of time of $D$ according to the analysis above, the total time of idle slot is

$$
T_{\text {slot }}=\left(E\left[N_{c}\right]+n\right) E\left[N_{\text {slot }}\right] \sigma=\frac{1-\tau}{\tau} \sigma
$$

Using equation (14)-(17), (21) and (24), we can get the medium access delay as

$$
D=n T_{s}+\frac{1-(1-\tau)^{n}-n \tau(1-\tau)^{n-1}}{\tau(1-\tau)^{n-1}} T_{c}+\frac{1-\tau}{\tau} \sigma
$$




\section{Numerical Results}

We assume each station has enough data to send to obtain the saturation throughput with the new backoff scheme. We change the number of stations to check the effect of throughput degradation due to increased collision probability.

All the parameters used in the analysis follow the same parameters in [11] for DSSS summarized in Table I. We assume the application data payload is 1000 bytes, IP header and UDP header are 20 and 8 bytes, respectively. Thus the packet payload at MAC layer becomes 1028 bytes.

Table 1. System parameters for MAC and DSSS PHY Layer

\begin{tabular}{|l|l|}
\hline Packet payload & 8224 bits \\
\hline MAC header & 224 bits \\
\hline PHY header & 192 bits \\
\hline ACK & 112 bits + PHY header \\
\hline RTS & 160 bits + PHY header \\
\hline CTS & 112 bits + PHY header \\
\hline Channel bit rate & $1 \mathrm{Mbps}$ \\
\hline Propagation delay & $1 \mathrm{us}$ \\
\hline Slot time & $20 \mathrm{us}$ \\
\hline SIFS & $10 \mathrm{us}$ \\
\hline DIFS & $50 \mathrm{us}$ \\
\hline
\end{tabular}

Fig. 5 shows the results of basic access method. From the figure, observe that the proposed scheme shows better saturation throughput than that of [11]. The results with RTS/CTS access method are similar to those of basic access method as shown in Fig 6. Note that the scale of vertical axis of Fig. 6 is slightly different from that of Fig. 5. From the comparisons, the proposed scheme is verified to reduce the chance of collision.

Fig. 7 shows that the delay of the basic access method highly depends on the initial contention window size, W, while Fig. 8 shows that RTS/CTS access method does not. In addition, the proposed scheme allows lower delay than [11]. The improvement in the delay is due to reduced collision probability between active stations and collided stations by using the proposed backoff time counter scheme.

Fig. 9 and 10 show the delay versus the number of wireless stations for the basic access method and RTS/CTS access method, respectively. The initial contention window size is set to 32. The delay for the basic access method is larger than that for the RTS/CTS access method under the same condition. This may be caused by the lager collision delay in basic access method. Note that delay for both the access methods strongly depends on the number of stations in the network. 


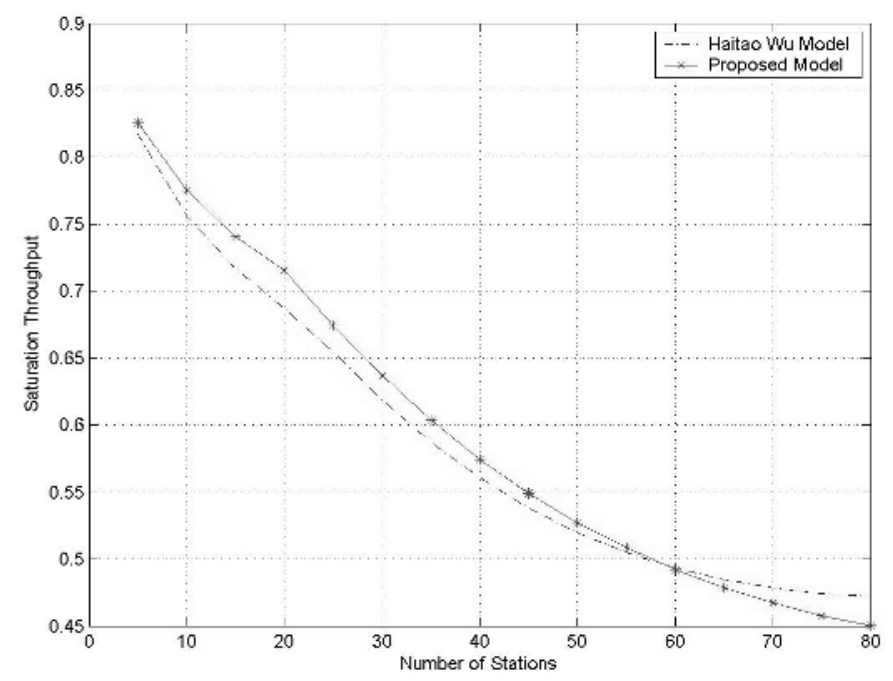

Fig. 5. Throughput: basic access method.

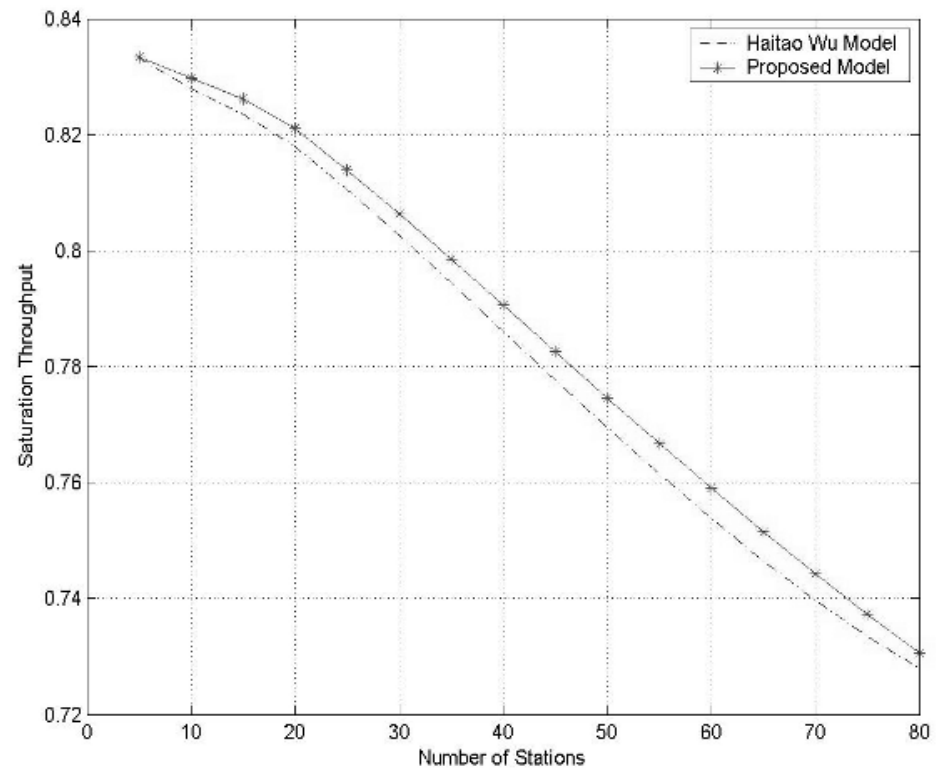

Fig. 6. Throughput: RTS/CTS access method. 


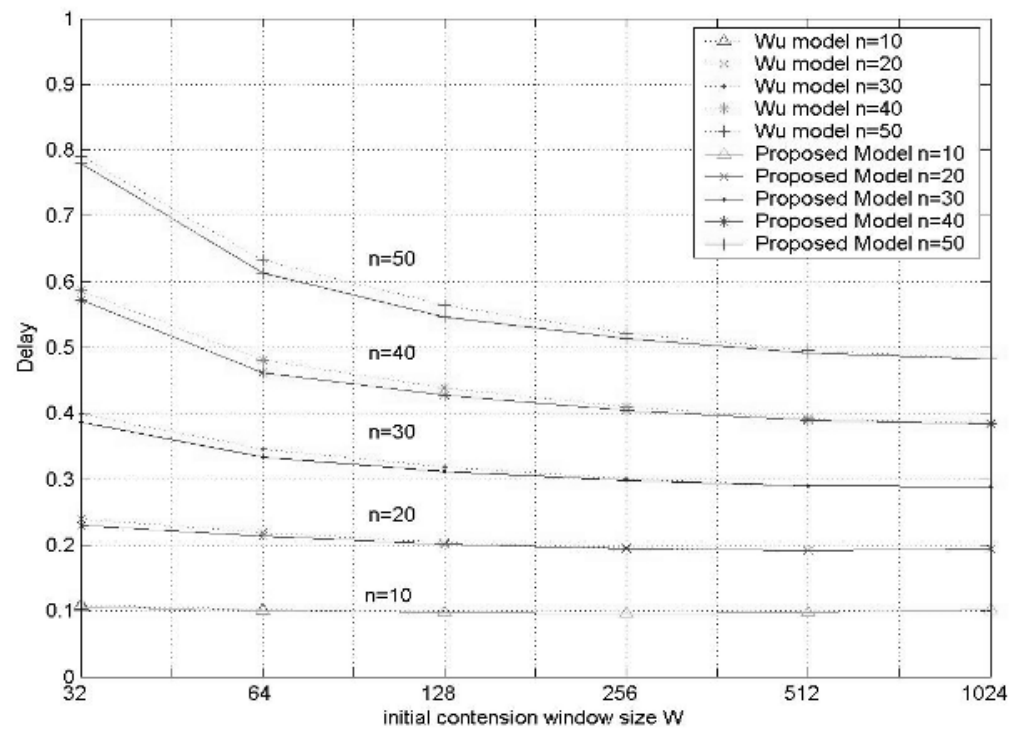

Fig. 7. Delay verse initial contention window size W for basic access method.

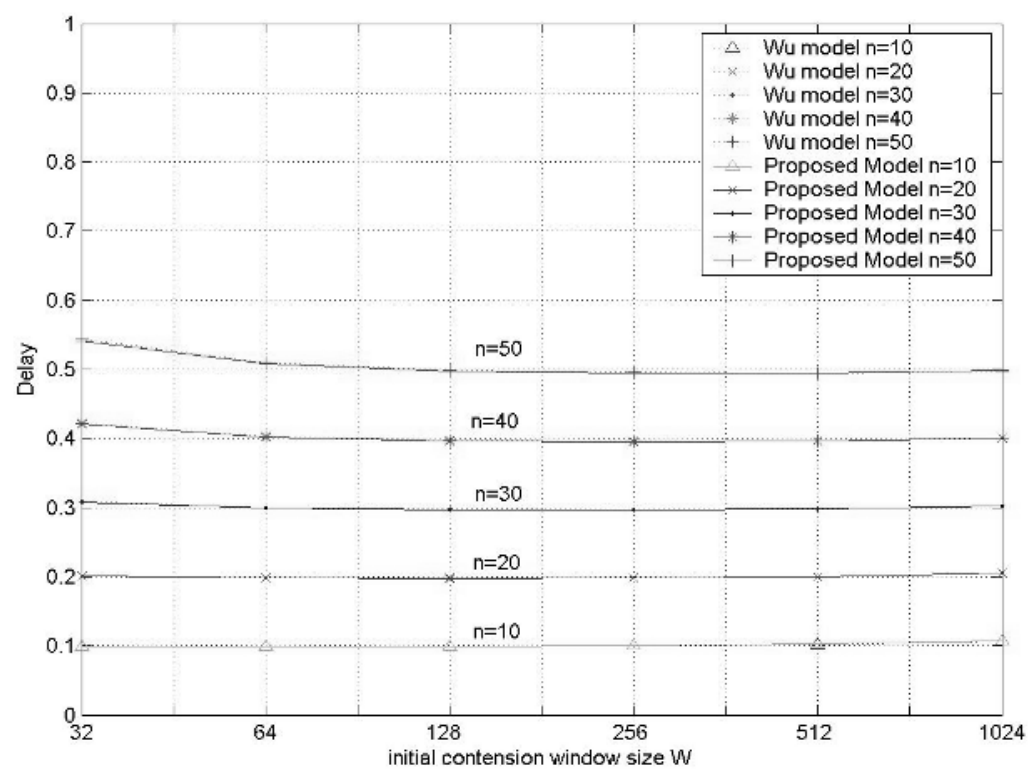

Fig. 8. Delay verse initial contention window size W for RTS/CTS access method. 


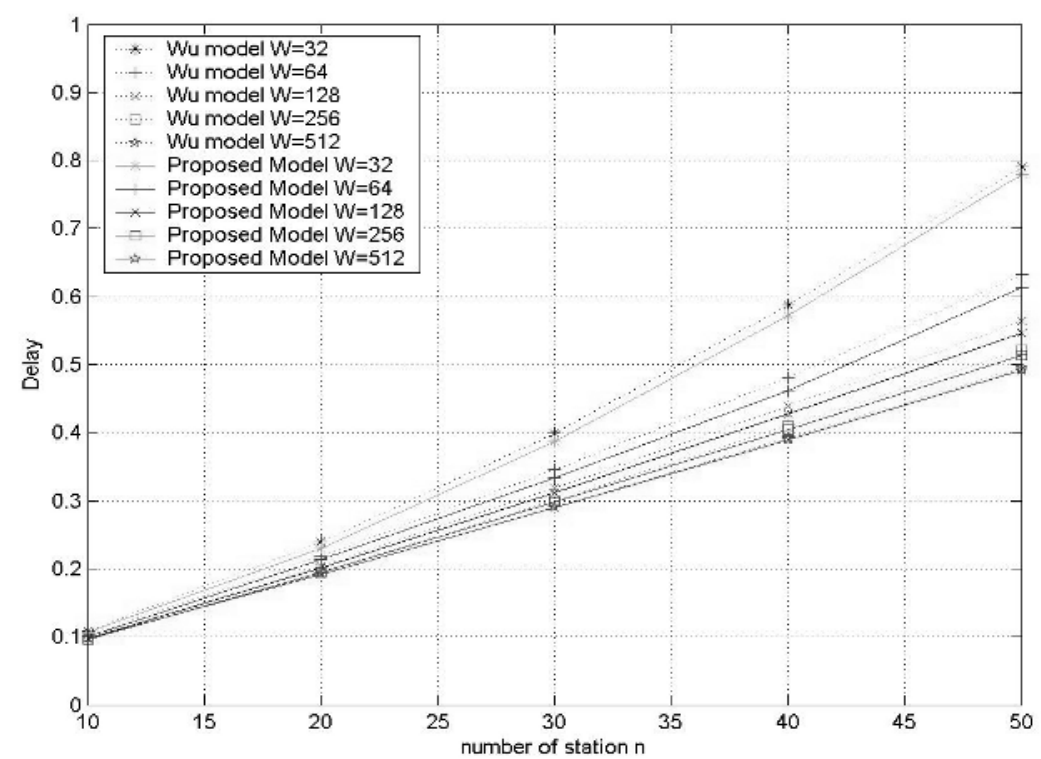

Fig. 9. Delay verse the number of stations for basic access method

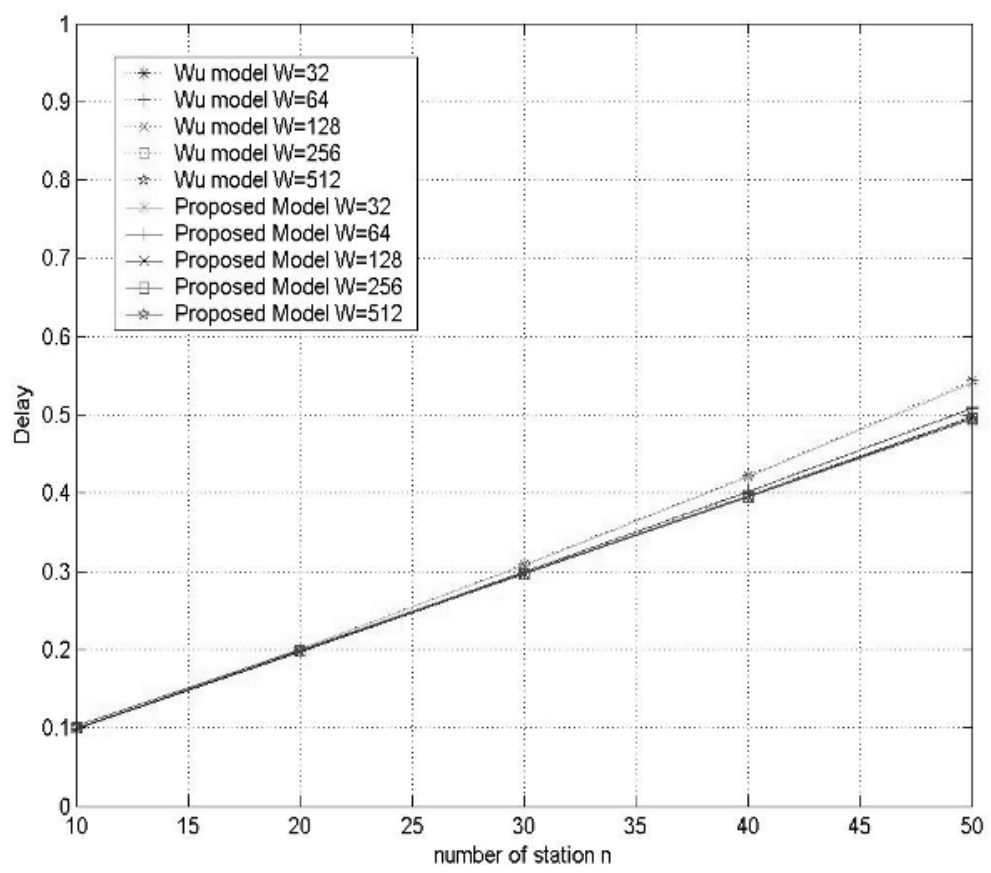

Fig. 10. Delay verse the number of stations for RTS/CTS access method 


\section{Conclusions}

This paper has proposed a new backoff scheme for CSMA/CA protocols, analyzed the throughput and delay, and based on a bi-dimensional discrete Markov chain. The analytical results showed that the proposed scheme allows higher throughput and lower delay compared to the legacy mechanism [11] at high traffic conditions. Comparing with the model in [11], even though the proposed scheme shows better performance, fairness remains to be solved. The reason for unfairness in the new scheme is due to the fact that the selection is not made within the full range of the congestion window. Future works will be focused on the enhancement of the proposed scheme and the corresponding analytical model by guaranteeing the fairness.

\section{References}

[1] IEEE standard for Wireless LAN Medium Access Control (MAC) and Physical Layer (PHY) specifications, ISO/IEC 8802-11: 1999 Edition, Aug. 1999

[2] B. P. Crow, J. G. Kim, et. "IEEE 802.11 Wireless Local Area Networks", IEEE Communication magazine. Sept. 1997

[3] J. Weinmiller, M. Schlager, A. Festag and A. Wolisz, "Performance study of access control in wireless LANs IEEE 802.11 DFWMAC and ETSI RES 10 HIPERLAN", Mobile Networks and Applications, pp. 55-67, Vol. 2, 1997

[4] F. Cali, M. Conti and E. Gregori, "IEEE 802.11 wireless LAN: Capacity analysis and protocol enhancement", INFOCOM'98, 1998

[5] H. S. Chhaya and S. Gupta, "Performance modeling of asynchronous data transfer methods of IEEE 802.11 MAC protocol", Wireless Networks, pp. 217-234, Vol. 3, 1997

[6] G. Bianchi, "IEEE 802.11 - Saturation Throughput Analysis", IEEE Communications Letters, pp. 318-320, Vol. 2, No. 12, Dec. 1998

[7] G. Bianchi, L. Fratta and M. Oliveri, "Performance analysis of IEEE 802.11 CSMA/CA medium access control protocol”, Proc. IEEE PIMRC, pp. 407-411. Oct. 1996

[8] G. Bianchi, "Performance Analysis of the IEEE 802.11 Distributed Coordination Function", IEEE Journal on Selected Area in Communication. pp. 514-519, Vol. 18, No. 3, Mar. 2000

[9] K. C. Huang K. C. Chen, "Interference analysis of nonpersistent CSMA with hidden terminals in multicell wireless data networks", Proc. IEEE PIMRC, pp. 907-911. Sep. 1995

[10] H. Wu, Y. Peng, K. Long and S. Cheng, "A Simple model of IEEE 802.11 Wireless LAN", Proceedings. ICII 2001 - Beijing. 2001

[11] H. Wu, Y. Peng, K. Long and J. Ma, "Performance of reliable transport protocol over IEEE 802.11 wireless LAN: analysis and enhancement”, Proc. IEEE Inforcom'02. 2002

[12] G. Wang, Y. Shu, and L. Zhang, "Delay Analysis of the IEEE 802.11 DCF", Proc. IEEE PIMRC, pp. 1737-1741, 2003

[13] K. H. Choi, K.S. Jang and D.R. Shin, "Delay and Collision Reduction Mechanism for Distributed Fair Scheduling in Wireless LANs", ICCSA 2004 Italy Perugia

[14] H. J. Shin and D. R. Shin, "Throughput Analysis Based on a New Backoff Scheme", CIC2004, pp. 79-82, LasVegas USA, 2004 